\title{
Estabilidade de componentes bioativos do suco tropical de goiaba não adoçado obtido pelos processos de enchimento a quente e asséptico
}

\author{
Stability of bioactive compounds of unsweetened tropical guava juice obtained by hot fill and aseptic processes
}

Daniele Sales da SILVA ${ }^{1}$, Geraldo Arraes MAIA ${ }^{1 \star}$, Paulo Henrique Machado de SOUSA ${ }^{1}$, Raimundo Wilane de FIGUEIREDO ${ }^{1}$, José Maria Correia da COSTA ${ }^{1}$, Ana Valquíria Vasconcelos da FONSECA ${ }^{1}$

\begin{abstract}
Resumo
A goiaba é um dos frutos tropicais de maior valor nutricional, rico em vitamina C e pró-vitamina A, possuindo uma excelente aceitação para consumo in natura, como também uma vasta aplicação industrial. A vitamina $\mathrm{C}$, os carotenoides, as antocianinas e os compostos fenólicos são considerados substâncias com atividade antioxidante, e seu consumo regular tem ação protetora efetiva contra os processos oxidativos que naturalmente ocorrem no organismo. Este trabalho objetivou estudar a estabilidade da vitamina C, carotenoides totais, antocianinas totais e compostos fenólicos totais do suco tropical de goiaba não adoçado obtido pelos processos de enchimento a quente e asséptico durante 250 dias de armazenamento à temperatura ambiente $\left(28 \pm 2{ }^{\circ} \mathrm{C}\right)$. Os resultados demonstraram que os conteúdos de carotenoides totais e os fenólicos totais não apresentaram variação durante o armazenamento, enquanto que a vitamina $\mathrm{C}$ apresentou redução.

Palavras-chave: Psidium guajava; qualidade; vitamina C; carotenoides; antocianinas; fenólicos totais.
\end{abstract}

\begin{abstract}
The guava is one of the tropical fruits with the highest nutritional value, rich in vitamins $\mathrm{C}$ and pro-vitamin $\mathrm{A}$, possessing excellent acceptance of in natura consumption as well as great industrial applicability. Vitamin C, carotenoids, anthocyanins and phenolics are considered compounds with antioxidant activity and the consumption of these substances in the daily diet can produce an effective protective action against the oxidative processes that occur naturally in the organism. This work aimed to study the stability of the vitamin C, carotenoids, anthocyanins and total phenolics of unsweetened guava tropical juice obtained by hot fill and aseptic processes for 250 days of storage at room temperature $\left(28 \pm 2{ }^{\circ} \mathrm{C}\right)$. The results showed that the total carotenoids and total phenolics did not vary during the storage time, while the vitamin $\mathrm{C}$ decreased.
\end{abstract}

Keywords: Psidium guajava; quality; vitamin C; carotenoids; anthocyanins; total phenolics.

\section{Introdução}

A goiaba é um dos frutos de maior importância nas regiões tropicais e subtropicais, não só devido ao seu elevado valor nutritivo, mas também pela excelente aceitação para consumo in natura, pela capacidade de desenvolvimento em condições adversas e pela grande aplicação industrial. Nesse sentido, além do seu consumo in natura, ela é utilizada na fabricação de doces, compotas, geleias, frutas em calda, purês, alimentos para crianças, xaropes, fermentados, entre outros produtos. Ao natural, a goiaba contém bastante vitamina $\mathrm{C}$, quantidades razoáveis de pró-vitamina A e vitaminas do complexo $\mathrm{B}$, e sais minerais como cálcio, fósforo e ferro (BRASIL; MAIA; FIGUEIREDO, 1996; MAIA et al., 2002; BRUNINI; OLIVEIRA; VARANDA, 2003).

Segundo a tabela da USDA (2006), a goiaba contém quatro vezes mais vitamina $C$ do que a laranja, que possui $50 \mathrm{mg}$ de vitamina C. $100 \mathrm{~g}^{-1}$, além de ser fonte de licopeno, seu teor é duas vezes maior do que o presente no tomate.

Os carotenoides compõem um grupo de compostos responsáveis pelas cores amarela, laranja e vermelha de muitos alimentos de origem vegetal e alguns de origem animal. São também citados como responsáveis pela diminuição do risco de doenças como câncer e doenças cardiovasculares (MATIOLI; RODRIGUEZ-AMAYA, 2003).

A vitamina $C$ desempenha várias funções biológicas relacionadas ao sistema imune, formação de colágeno, absorção de ferro, inibição da formação de nitrosaminas e atividade antioxidante (VANNUCHI; JORDÃO Jr., 1998), além de facilitar o uso do cálcio na construção dos ossos e vasos sanguíneos (WTCR/AICR, 1997).

As frutas, principalmente as que apresentam a coloração vermelha/azul, são as mais importantes fontes de compostos fenólicos em dietas alimentares. Muitos destes compostos apresentam vários efeitos biológicos, incluindo ações antioxidante, antimicrobiana, anti-inflamatória e vasodilatadora. Estes compostos fenólicos apresentam diversas funções de defesa para as plantas, não somente contra agentes do ambiente (luz, temperatura e umidade), mas também para fatores internos, incluindo diferenças genéticas, nutrientes, hormônios,

Recebido para publicação em 24/4/2008

Aceito para publicação em 3/1/2009 (003464)

${ }^{1}$ Departamento de Tecnologia de Alimentos, Universidade Federal do Ceará, Av. Mister Hull, 2977, CP 12168, CEP 60356-000, Campus do Pici, Fortaleza, Ceará, Brasil,

E-mail:gmaia@secrel.com.br

${ }^{*}$ A quem a correspondência deve ser enviada 
contribuindo para a sua síntese (AHERNE; O'BRIEN, 2002; SELLAPPAN; AKOH; KREWER, 2002).

Dentre os compostos fenólicos com propriedade antioxidante, destacam-se os flavonoides, que quimicamente englobam as antocianinas e os flavonóis. As antocianinas são pigmentos solúveis em água, amplamente difundidas no reino vegetal e conferem as várias nuanças de cores entre laranja, vermelha e azul encontradas em frutas, vegetais, flores, folhas e raízes (FRANCIS, 1989).

Um grande interesse pelas antocianinas vem sendo demonstrado pelas observações promissoras de seu potencial benéfico à saúde, decorrente de sua ação antioxidante (VENDRAMINI; TRUGO, 2004).

O prazo de vida comercial é o período de tempo decorrido entre a produção e o consumo de um produto alimentício, no qual a aceitabilidade do produto pelo consumidor é mantida e verifica-se no produto um nível satisfatório de qualidade. Esta qualidade pode ser avaliada por atributos sensoriais (sabor, cor, aroma, textura e aparência), pela carga microbiana, pela absorção de componentes da embalagem ou pelo valor nutricional (SARANTÓPOULOS; OLIVEIRA; CANAVESI, 2001).

Os métodos mais utilizados pelas indústrias para a preservação de sucos de frutas tropicais consistem nos processos de enchimento a quente (Hot Fill) e enchimento asséptico. No primeiro processo, o suco é submetido a um tratamento térmico de pasteurização, por meio de trocadores de calor, em temperatura acima de $90{ }^{\circ} \mathrm{C}$, durante 45 a 60 segundos, seguido de enchimento a quente imediatamente após a saída do trocador de calor e, em seguida, é resfriado até temperatura máxima de $37^{\circ} \mathrm{C}$. No processo asséptico, o produto é tratado termicamente, resfriado em seguida em trocadores de calor e flui para as unidades assépticas, nas quais é colocado em embalagens previamente esterilizadas, sem contato com o ar atmosférico ou qualquer fonte de contaminação (MAIA; SOUSA; LIMA, 2007).

Diante do exposto, este trabalho objetivou estudar a estabilidade da vitamina $\mathrm{C}$, carotenoides totais, antocianinas totais e compostos fenólicos totais no suco tropical de goiaba não adoçado obtido pelos processos de enchimento a quente e asséptico, armazenado durante 250 dias à temperatura ambiente $\left(28 \pm 2^{\circ} \mathrm{C}\right)$.

\section{Material e métodos}

\subsection{Matéria-prima}

Sucos tropicais de goiaba não adoçados obtidos pelos processos de enchimento a quente (embalagem de vidro de $500 \mathrm{~mL}$ ) e asséptico (embalagem cartonada de $1000 \mathrm{~mL}$ ) armazenados a $28 \pm 2{ }^{\circ} \mathrm{C}$.

\subsection{Processamento do suco de goiaba}

As goiabas foram colhidas manualmente, acondicionadas e, em seguida, transportadas para a unidade de processamento, onde foram pesadas. Os frutos foram selecionados e lavados por imersão em água clorada. Em seguida, foram passados por uma despolpadeira, onde se obteve a polpa refinada, a partir da qual se realizou a formulação (água, 50\% de polpa de goiaba, conservantes: benzoato de sódio e metabissulfito de sódio, acidulante: ácido cítrico), procedendo-se em seguida à homogeneização e posteriormente à desaeração. Na sequência, para a produção através do processo de enchimento a quente (Hot Fill), o suco foi submetido a tratamento térmico, a $90^{\circ} \mathrm{C}$ por 60 segundos, seguido de enchimento a $85^{\circ} \mathrm{C}$ em garrafas de vidro de $500 \mathrm{~mL}$ e fechamento imediato por tampas plásticas rosqueadas. Após o fechamento as garrafas foram resfriadas, acondicionadas em caixas de papelão e armazenadas à temperatura de $28 \pm 2{ }^{\circ} \mathrm{C}$ para estudo de estabilidade.

Os procedimentos para a elaboração do suco tropical de goiaba obtido pelo processo asséptico foram os mesmos descritos para o enchimento a quente até a etapa de tratamento térmico, sendo que, após o tratamento térmico, o suco foi resfriado no próprio trocador de calor e o enchimento realizado assepticamente a $25^{\circ} \mathrm{C}$ em embalagens cartonadas. Após o envase, as caixinhas foram acondicionadas em caixas de papelão e armazenadas a $28 \pm 2{ }^{\circ} \mathrm{C}$ para estudos de estabilidade (Figura 1).

\subsection{Determinações químicas e físico-químicas}

A vitamina $C$ foi determinada de acordo com Brasil (2005) adaptado, através de método titulométrico com solução de
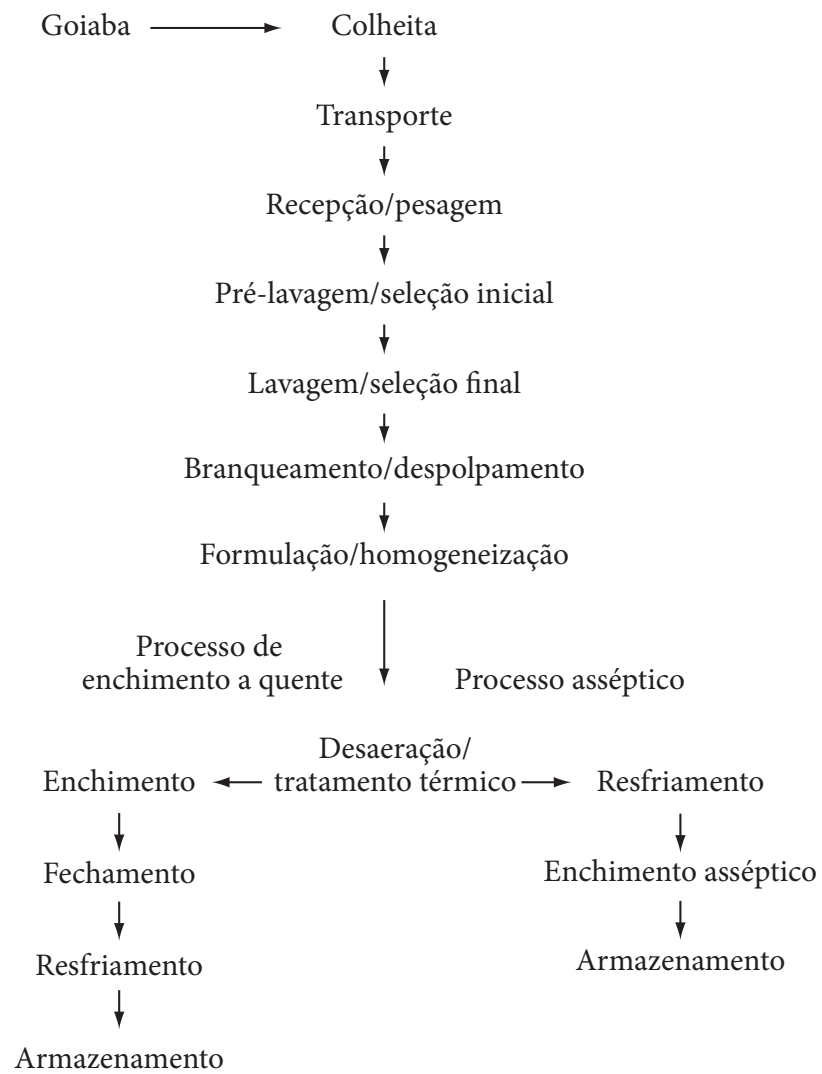

Figura 1. Produção do suco tropical de goiaba não adoçado obtido pelos processos de enchimento a quente e asséptico. 
2,6-Diclorofenol Indofenol (DCFI), utilizando ácido oxálico $1 \%$ para diluição das amostras, sendo os resultados expressos em miligrama de ácido ascórbico. $100 \mathrm{~mL}^{-1}$ de suco. Os carotenoides totais foram determinados segundo Higby (1962), sendo os resultados expressos em miligrama de carotenoides totais. $100 \mathrm{~mL}^{-1}$. A determinação de antocianinas totais foi realizada de acordo com o método de Francis (1982) adaptado. Foi feita uma extração através da homogeneização de $1 \mathrm{~mL}$ da amostra com solução de $\mathrm{HCl}(1,5 \mathrm{~N})$ e etanol $85 \%$ durante um período de 16 horas sob refrigeração $\left(\mathrm{T}=4 \pm 1{ }^{\circ} \mathrm{C}\right)$ e ao abrigo da luz, sendo os extratos filtrados e então realizada a leitura em espectrofotômetro da marca MICRONAL, modelo B582, com absorbância medida no comprimento de onda de $535 \mathrm{~nm}$. Os resultados foram expressos em miligrama de antocianinas totais. $100 \mathrm{~mL}^{-1}$. Os fenólicos totais foram determinados de acordo com a metodologia descrita por Zielinski e Kozlowska (2000), usando o reagente Folin-Ciocalteu e o ácido tânico como padrão, sendo os resultados expressos em miligrama de ácido tânico. $100 \mathrm{~mL}^{-1}$.

\subsection{Análise estatística}

$\mathrm{O}$ experimento foi conduzido segundo o delineamento em parcelas subdivididas, com dois tratamentos (processo de enchimento a quente e processo asséptico) nas parcelas e seis tempos de armazenamento $(0,50,100,150,200$ e 250 dias $)$ nas subparcelas em fatorial inteiramente ao acaso, com duas repetições de experimento.

Os resultados obtidos para os parâmetros estudados foram analisados estatisticamente por análise de variância (teste F) e de regressão e, quando conveniente, foi realizado teste de Tukey para comparação de médias, ao nível de $5 \%$ de probabilidade, através do programa estatístico SAS versão 9.1 (SAS INSTITUTE, 2006). A análise de regressão foi realizada até o modelo de segundo grau, testando-se a falta de ajuste e os coeficientes da equação através do teste de $T(\mathrm{p} \leq 0,05)$.

\section{Resultados e discussão}

Para todos os parâmetros analisados (vitamina C, carotenoides totais, antocianinas totais e fenólicos totais) não foram detectados efeitos significativos ( $p>0,05)$ da interação entre os tratamentos e tempo de armazenamento (Tabela 1). Com base nesses resultados, foram estudados os efeitos de tratamento e tempo de armazenamento separadamente. Somente se observou diferença estatística entre os tratamentos
( $p \leq 0,05)$ para fenólicos totais, enquanto que para o tempo de armazenamento, somente os parâmetros vitamina $\mathrm{C}$ e antocianinas ajustaram-se a um modelo de regressão, sendo representados por apenas uma equação, que designa os dois tratamentos (Tabela 1, Figuras 2 e 4).

A análise estatística dos valores médios obtidos para vitamina $\mathrm{C}$ em função do tempo de armazenamento apresentou diferença significativa $(\mathrm{p} \leq 0,05)$, mostrando que a regressão foi do tipo linear (Figura 2).

Os resultados obtidos oscilaram entre 22,25 e $38,41 \mathrm{mg}$ de ácido ascórbico.100 mL $\mathrm{mL}^{-1}$. De acordo com a legislação (BRASIL, 2003), o mínimo de ácido ascórbico permitido para o suco tropical de goiaba não adoçado é de $30 \mathrm{mg} .100 \mathrm{~mL}^{-1}$. Fernandes et al. (2006), estudando os parâmetros de identidade e qualidade para suco tropical de goiaba, verificaram valores variando entre 11,6 e $33,3 \mathrm{mg}$ de vitamina C. $100 \mathrm{~mL}^{-1}$. Suntornsuk et al. (2002) mostraram que o teor de vitamina C em suco de goiaba recém-preparado foi de $26,10 \mathrm{mg}$ de vitamina C. $100 \mathrm{~mL}^{-1}$ e que, para 7 e 14 dias de armazenamento, o suco apresentou teores de 25,06 e 24,45, respectivamente. Brasil, Maia e Figueiredo (1995), estudando a estabilidade do suco de goiaba clarificado por um período de 120 dias de armazenamento, encontraram valores de vitamina $\mathrm{C}$ oscilando entre 54,28 $58,74 \mathrm{mg} .100 \mathrm{~mL}^{-1}$.

Existe uma vasta literatura que comenta a respeito da oxidação química da vitamina C e/ou degradação térmica como consequência do branqueamento, cozimento, pasteurização, esterilização, desidratação e congelamento

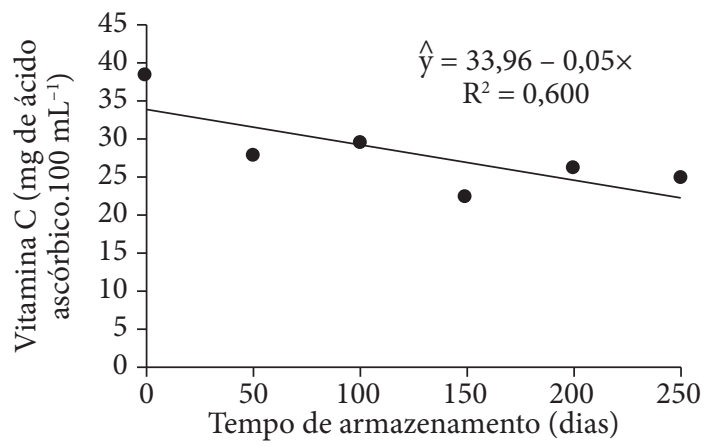

Figura 2. Média dos teores de vitamina C no suco tropical de goiaba não adoçado obtido pelos processos de enchimento a quente e asséptico, durante 250 dias à temperatura ambiente $\left(28 \pm 2^{\circ} \mathrm{C}\right)$.

Tabela 1. Resumo da variância para vitamina C, carotenóides totais, antocianinas totais e fenólicos totais presentes no suco tropical de goiaba não adoçado obtido pelos processos de enchimento a quente e asséptico.

\begin{tabular}{|c|c|c|c|c|c|}
\hline \multirow{2}{*}{$\begin{array}{l}\text { Fonte de } \\
\text { variação }\end{array}$} & \multirow[t]{2}{*}{ GL } & \multicolumn{4}{|c|}{ Quadrado médio } \\
\hline & & $\begin{array}{c}\text { Vitamina C } \\
\left(\mathrm{mg} .100 \mathrm{~mL}^{-1}\right)\end{array}$ & $\begin{array}{l}\text { Carotenóides } \\
\left(\mathrm{mg} .100 \mathrm{~mL}^{-1}\right)\end{array}$ & $\begin{array}{l}\text { Antocianinas } \\
\left(\mathrm{mg} .100 \mathrm{~mL}^{-1}\right)\end{array}$ & $\begin{array}{c}\text { Fenólicos totais } \\
\left(\mathrm{mg} \text { de ácido tânico. } 100 \mathrm{~mL}^{-1}\right)\end{array}$ \\
\hline Trat (A) & 1 & $135,7077^{\mathrm{ns}}$ & $0,0005^{\mathrm{ns}}$ & $0,0176^{\mathrm{ns}}$ & $4425,4504^{\star}$ \\
\hline Erro (A) & 118 & 106,8826 & 0,0307 & 0,0292 & 61,5177 \\
\hline Tempo (B) & 5 & $125,7889^{*}$ & $0,0187^{\mathrm{ns}}$ & $0,0405^{*}$ & $466,6152^{\mathrm{ns}}$ \\
\hline$\left(\mathrm{A}^{\star} \mathrm{B}\right)$ & 5 & $9,9308^{\mathrm{ns}}$ & $0,0333^{\text {ns }}$ & $0,0079^{\text {ns }}$ & $26,8414^{\mathrm{ns}}$ \\
\hline Erro $\left(A^{*} B\right)$ & 586 & 5,1253 & 0,0121 & 0,0023 & 12,8039 \\
\hline
\end{tabular}

${ }^{\star}$ Significativo ao nível de $5 \%$ de probabilidade $(\mathrm{p} \leq 0,05) ;{ }^{\text {ns }}$ não significativo ao nível de $5 \%$ de probabilidade $(\mathrm{p}>0,05)$; GL - grau de liberdade. 
(SAHARI; BOOSTANI; HAMIDI, 2004; JOHNSTON; HALE, 2005; POLYDERA; STOFOROS; TAOUKIS, 2005; VIKRAM; RAMESH; PRAPULLA, 2005; BURDURLU; KOCA; KARADENIZ, 2006). Uma causa adicional da redução da vitamina C é sua participação na reação de Maillard (DJILAS; MILIC, 1994). Segundo Martin et al. (1995), a luz tem um efeito significativo sobre a destruição da vitamina $\mathrm{C}$ em suco de laranja pasteurizado e envasado a quente, o que demonstra o efeito catalítico da luz sobre a oxidação da vitamina C.

Na Tabela 2, estão apresentados os valores obtidos das médias da vitamina $\mathrm{C}$ para os processos de enchimento a quente e asséptico durante os 250 dias de armazenamento.

Os valores das médias observados mostram que o suco envasado pelo processo asséptico preservou melhor o teor de vitamina C em comparação ao enchimento a quente. Resultados similares foram relatados por Mannheim e Havkin (1981) em suco de laranja.

Comparando-se os resultados obtidos de vitamina $\mathrm{C}$ para os dois processos analisados, após 250 dias de armazenamento, notase uma redução do teor em ambos, sendo que para o enchimento a quente a perda foi de $36,3 \%$ e para o asséptico de $34,9 \%$. O menor teor de vitamina $\mathrm{C}$ para o processo de enchimento a quente pode estar relacionado à influência da temperatura, ao tempo de exposição ao calor durante o processamento e também pela influência da natureza da embalagem.

Freitas et al. (2006a), estudando a estabilidade do suco integral de acerola não adoçado e envasado pelo processo de enchimento a quente durante 350 dias de armazenamento, verificaram uma redução no teor de ácido ascórbico de $45,12 \%$ ao final do armazenamento. Já em um estudo com suco tropical de acerola adoçado, Freitas et al. (2006b) concluíram que a vitamina $\mathrm{C}$ decresceu com o armazenamento em ambos os processos, sendo que a perda para o enchimento a quente foi de $23,61 \%$ e para o asséptico foi de $35,95 \%$, após 350 dias de armazenamento.

Estudando a estabilidade do suco tropical de manga adoçado e envasado pelos processos de enchimento a quente e asséptico durante 350 dias de armazenamento, Magalhães (2005) verificou uma redução no teor de vitamina C com o armazenamento em ambos os processos, sendo de $34,29 \%$ para o enchimento a quente e de $75,03 \%$ para o processo asséptico.

Tabela 2. Teores de vitamina $\mathrm{C}$ no suco tropical de goiaba não adoçado obtido pelos processos de enchimento a quente e asséptico durante 250 dias a temperatura ambiente $\left(28 \pm 2{ }^{\circ} \mathrm{C}\right)$ (médias \pm desvio padrão) $(\mathrm{n}=3)$.

\begin{tabular}{ccc}
\hline \multirow{2}{*}{$\begin{array}{c}\text { Tempo de } \\
\text { armazenamento(dias) }\end{array}$} & \multicolumn{2}{c}{ Vitamina C $\left(\mathrm{mg} .100 \mathrm{~mL}^{-1}\right)$} \\
\hline 0 & Enchimento a quente & Processo asséptico \\
\hline 50 & $24,9 \pm 0,0$ & $43,0 \pm 1,8$ \\
100 & $28,7 \pm 1,2$ & $31,4 \pm 4,4$ \\
150 & $20,9 \pm 1,8$ & $30,5 \pm 8,6$ \\
200 & $25,3 \pm 2,4$ & $23,6 \pm 3,7$ \\
250 & $21,6 \pm 3,4$ & $27,0 \pm 9,6$ \\
\hline
\end{tabular}

Costa et al. (2003) verificaram uma redução no teor de vitamina C de $25,65 \%$ em suco tropical de caju obtido pelo processo de enchimento a quente ao final de 350 dias de armazenamento, enquanto que para o processo asséptico a perda foi de $26,74 \%$.

Os teores de carotenoides totais não apresentaram variação significativa ao longo do armazenamento, sendo representados por uma reta constante na Figura 3, com valor médio de $1,08 \mathrm{mg}$ de carotenoides totais. $100 \mathrm{~mL}^{-1}$ de suco, para os dois tratamentos.

Na Tabela 3 estão apresentados os valores obtidos das médias dos carotenoides totais para os processos de enchimento a quente e asséptico durante os 250 dias de armazenamento.

Comparando-se os teores de carotenoides totais para os processos avaliados, observa-se uma redução para o enchimento a quente, no entanto, para o processo asséptico verifica-se um ligeiro aumento, em que inicialmente se obteve um teor de $0,93 \mathrm{mg}$ de carotenoides totais. $100 \mathrm{~mL}^{-1}$, e chegando-se, ao tempo 250 dias, a $1,14 \mathrm{mg}$ de carotenoides totais. $100 \mathrm{~mL}^{-1}$.

Freitas et al. (2006a) verificaram redução de 3,61\% no teor de carotenoides totais em relação ao início do armazenamento após 350 dias de armazenamento de um suco integral de acerola não adoçado.

Freitas et al. (2006b), em estudo da estabilidade do suco tropical de acerola adoçado envasado pelos processos

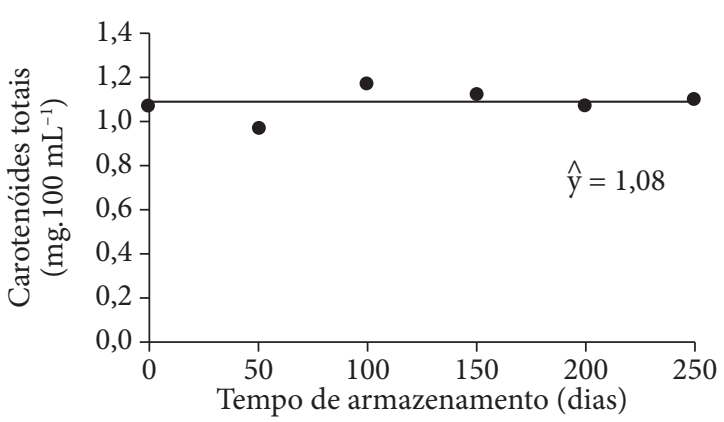

Figura 3. Média dos teores de carotenoides totais no suco tropical de goiaba não adoçado obtido pelos processos de enchimento a quente e asséptico durante 250 dias à temperatura ambiente $\left(28 \pm 2{ }^{\circ} \mathrm{C}\right)$.

Tabela 3. Teores de carotenóides totais no suco tropical de goiaba não adoçado obtido pelos processos de enchimento a quente e asséptico durante 250 dias a temperatura ambiente $\left(28 \pm 2{ }^{\circ} \mathrm{C}\right.$ ) (médias \pm desvio padrão) $(\mathrm{n}=3)$.

\begin{tabular}{ccc}
\hline Tempo de & \multicolumn{2}{c}{ Carotenóides totais $\left(\mathrm{mg} \cdot 100 \mathrm{~mL}^{-1}\right)$} \\
\cline { 2 - 3 } armazenamento (dias) & Enchimento a quente & Processo asséptico \\
\hline 0 & $1,20 \pm 0,01$ & $0,93 \pm 0,08$ \\
50 & $1,01 \pm 0,01$ & $0,93 \pm 0,28$ \\
100 & $1,24 \pm 0,23$ & $1,11 \pm 0,11$ \\
150 & $1,00 \pm 0,06$ & $1,24 \pm 0,03$ \\
200 & $1,03 \pm 0,11$ & $1,12 \pm 0,09$ \\
250 & $1,06 \pm 0,06$ & $1,14 \pm 0,01$ \\
\hline
\end{tabular}


de enchimento a quente e asséptico durante 350 dias de armazenamento, comparando os teores de carotenoides totais obtidos nos tempos zero e 350 dias, observaram para o processo de enchimento a quente uma redução da ordem de $12,5 \%$, enquanto que para o processo asséptico os valores apresentaram pouca variação nos tempos zero, 150, 200 e 350 dias $\left(0,11-0,16 \mathrm{mg}\right.$ de carotenoides totais. $\left.100 \mathrm{~mL}^{-1}\right)$.

A análise estatística dos valores obtidos para antocianinas totais apresentou correlação significativa em função do tempo de armazenamento $(\mathrm{p} \leq 0,05)$, apresentando comportamento linear (Figura 4). Os resultados oscilaram entre 0,22-0,49 mg.100 mL $\mathrm{m}^{-1}$ da amostra.

$\mathrm{Na}$ Tabela 4 estão apresentados os valores obtidos das médias das antocianinas totais para os processos de enchimento a quente e asséptico durante os 250 dias de armazenamento.

Comparando-se os dois processos, verificou-se que não houve perda dos conteúdos de antocianinas totais. Observou-se um valor de $0,53 \mathrm{mg}$ de antocianinas totais. $100 \mathrm{~mL}^{-1}$ para o processo de enchimento a quente ao final do armazenamento, enquanto que para o processo asséptico verificou-se um teor de $0,46 \mathrm{mg}$ de antocianinas totais. $100 \mathrm{~mL}^{-1}$ após o mesmo período.

Freitas et al. (2006b), em estudo de estabilidade do suco tropical de acerola adoçado envasado pelos processos de enchimento a quente e asséptico, encontraram valores similares de antocianinas totais nos tempos zero e 350 dias (0,41 mg de

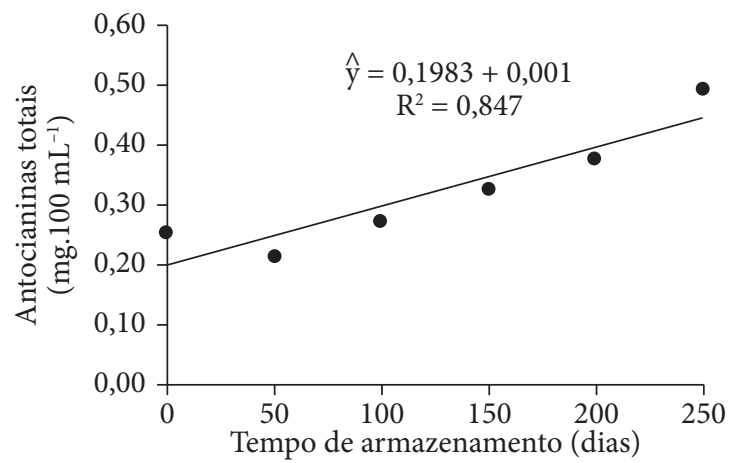

Figura 4. Média dos teores de antocianinas totais no suco tropical de goiaba não adoçado obtido pelos processos de enchimento a quente e asséptico durante 250 dias à temperatura ambiente $\left(28 \pm 2^{\circ} \mathrm{C}\right)$.

Tabela 4. Teores de antocianinas totais no suco tropical de goiaba não adoçado obtido pelos processos de enchimento a quente e asséptico durante 250 dias a temperatura ambiente $\left(28 \pm 2{ }^{\circ} \mathrm{C}\right.$ ) (médias \pm desvio padrão) $(\mathrm{n}=3)$.

\begin{tabular}{ccc}
\hline \multirow{2}{*}{$\begin{array}{c}\text { Tempo de } \\
\text { armazenamento (dias) }\end{array}$} & \multicolumn{2}{c}{ Antocianinas totais (mg.100 $\left.\mathrm{mL}^{-1}\right)$} \\
\hline 0 & Enchimento a quente & Processo asséptico \\
\hline 50 & $0,33 \pm 0,07$ & $0,18 \pm 0,11$ \\
100 & $0,26 \pm 0,04$ & $0,18 \pm 0,11$ \\
150 & $0,32 \pm 0,01$ & $0,23 \pm 0,04$ \\
200 & $0,35 \pm 0,02$ & $0,31 \pm 0,15$ \\
250 & $0,32 \pm 0,01$ & $0,43 \pm 0,00$ \\
\hline
\end{tabular}

antocianinas. $100 \mathrm{~mL}^{-1}$ ) obtidos para o processo de enchimento a quente; não sendo observadas perdas ao final do período de armazenamento. Todavia, para o processo asséptico constatouse, ao final do tempo de 350 dias, uma redução de $86,89 \%$ em relação ao tempo inicial.

Os valores encontrados para compostos fenólicos totais não apresentaram diferença significativa no decorrer do armazenamento ( $\mathrm{p}>0,05$ ), cujos teores variaram de 84,68 a 112,44 mg de ácido tânico.100 $\mathrm{mL}^{-1}$ da amostra (Figura 5).

A quantificação dos compostos fenólicos em sucos de frutos tem a finalidade de avaliar o potencial de escurecimento durante ou após o processamento e também a possibilidade de interferência desses compostos no sabor, devido à característica de adstringência de alguns deles (FILGUEIRAS; ALVES; MOURA, 2000). Além disso, os compostos fenólicos são poderosos antioxidantes e, portanto, têm elevado apelo funcional.

Brasil, Maia e Figueiredo (1995), estudando as mudanças físico-químicas durante a extração e a clarificação de suco de goiaba, encontraram valores de 51,5 e 41,8 mg de taninos.100 $\mathrm{g}^{-1}$, para suco clarificado de goiaba e suco clarificado de goiaba envasado por processo hot pack, respectivamente.

Aplicando-se o teste de Tukey ao nível de 5\% de probabilidade, nota-se que as médias gerais de fenólicos totais detectadas nos tratamentos apresentaram maiores valores para o processo de enchimento a quente (Tabela 5).

$\mathrm{Na}$ Tabela 6 estão apresentados os valores obtidos das médias dos fenólicos totais para os processos de enchimento a quente e asséptico durante os 250 dias de armazenamento.

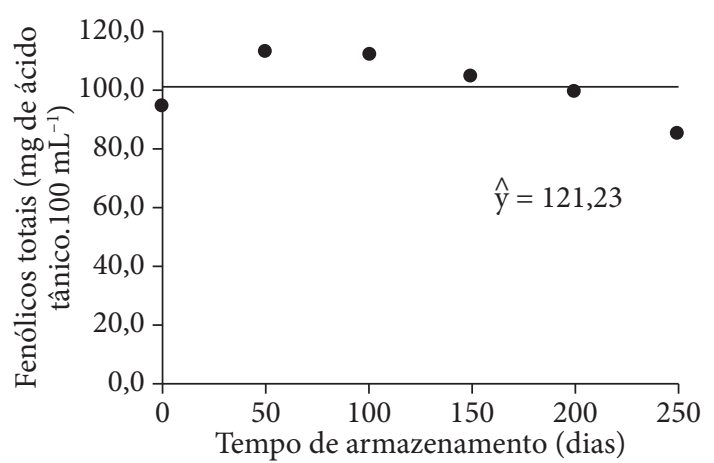

Figura 5. Média dos teores de fenólicos totais no suco tropical de goiaba não adoçado obtido pelos processos de enchimento a quente e asséptico, durante 250 dias à temperatura ambiente $\left(28 \pm 2{ }^{\circ} \mathrm{C}\right)$.

Tabela 5. Média dos fenólicos totais para o suco tropical de goiaba não adoçado obtido pelos processos de enchimento a quente e asséptico $\left(28 \pm 2^{\circ} \mathrm{C}\right)$.

\begin{tabular}{ll}
\hline \multicolumn{1}{c}{ Tratamentos } & \multicolumn{1}{c}{$\begin{array}{c}\text { Fenólicos totais } \\
\left(\mathrm{mg} \text { de ácido tânico. } 100 \mathrm{~mL}^{-1}\right)^{*}\end{array}$} \\
\hline Processo de Enchimento a quente & $114,81^{\mathrm{a}}$ \\
Processo Asséptico & $87,65^{\mathrm{b}}$ \\
\hline * Resultados com a mesma letra não apresentam diferença significatica $(\mathrm{p}>0,05)$ pelo \\
teste de Tukey.
\end{tabular}


Tabela 6. Teores de fenólicos totais no suco tropical de goiaba não adoçado obtido pelos processos de enchimento a quente e asséptico, durante 250 dias à temperatura ambiente $\left(28 \pm 2{ }^{\circ} \mathrm{C}\right.$ ) (médias \pm desvio padrão) $(\mathrm{n}=3)$.

\begin{tabular}{ccc}
\hline $\begin{array}{c}\text { Tempo de } \\
\text { armazenamento (dias) }\end{array}$ & \multicolumn{2}{c}{$\begin{array}{c}\text { Compostos fenólicos totais } \\
\text { (mg de ácido tânico.100 } \mathrm{mL}^{-1} \text { ) }\end{array}$} \\
\cline { 2 - 3 } & Enchimento a quente & Processo asséptico \\
\hline Zero & $110,15 \pm 6,29$ & $77,93 \pm 2,09$ \\
50 & $128,33 \pm 2,09$ & $96,55 \pm 3,39$ \\
100 & $126,60 \pm 4,53$ & $97,38 \pm 4,14$ \\
150 & $118,93 \pm 0,95$ & $90,73 \pm 2,51$ \\
200 & $109,90 \pm 6,08$ & $88,98 \pm 3,22$ \\
250 & $94,98 \pm 9,65$ & $74,38 \pm 2,44$ \\
\hline
\end{tabular}

Observou-se um decréscimo no teor de fenólicos totais para os dois processos avaliados tomando-se por base os tempos inicial e final de armazenamento. Para o suco obtido pelo processo de envase asséptico, foi verificado um menor teor, tornando-se necessário avaliar posteriormente a permeabilidade da embalagem ao oxigênio e a suposta ação de possíveis resíduos de peróxido de hidrogênio na embalagem.

\section{Conclusões}

Ambos os processos estudados (enchimento a quente e asséptico) de processamento de suco tropical de goiaba apresentaram boa estabilidade de seus componentes bioativos.

Os teores de vitamina $\mathrm{C}$ decresceram com o tempo de armazenamento em ambos os processos, chegando ao final do armazenamento com perdas similares: $36,3 \%$ para o processo de enchimento a quente e $34,9 \%$ para o asséptico.

Os valores de carotenoides totais não diferiram significativamente com o armazenamento, apresentando pouca variação nos sucos submetidos aos dois tipos de embalagens.

O teor de compostos fenólicos totais apresentou durante o armazenamento uma redução tanto para o processo de enchimento a quente como para o processo asséptico, no entanto, esse decréscimo foi mais intenso para o processo asséptico.

\section{Agradecimentos}

À Fundação Cearense de Apoio ao Desenvolvimento Científico e Tecnológico (FUNCAP) e ao CNPq pela concessão de bolsas de estudo e financiamento da pesquisa.

\section{Referências bibliográficas}

AHERNE, S. A.; O'BRIEN, N. M. Dietary flavonols: chemistry, food content, and, metabolism. Nutrition, v. 18, n. 1, p. 75-81, 2002.

BRASIL, I. M.; MAIA, G. A.; FIGUEIREDO, R. W. Physicochemical changes during extraction and clarification of guava juice. Food Chemistry, v. 54, n. 4, p. 383-386, 1995.
BRASIL, I. M.; MAIA, G. A.; FIGUEIREDO, R. W. Estudo do rendimento do suco de goiaba extraído por tratamento enzimático. Ciência e Tecnologia de Alimentos, v. 16, n. 1, p. 57-61, 1996.

BRASIL. Ministério da Agricultura, Pecuária e Abastecimento. Instrução Normativa n. 12, de 4 de setembro de 2003. Regulamento técnico para fixação dos padrões de identidade e qualidade gerais para suco tropical e de outras providências. Diário Oficial da República Federativa do Brasil, Poder Executivo, Brasília, DF, 09 set. 2003.

BRASIL. Ministério da Saúde. Métodos físico-químicos para análise de alimentos. Brasília, 2005. 1018 p.

BRUNINI, M. A.; OLIVEIRA, A. L.; VARANDA, D. B. Avaliação da qualidade de polpa de goiaba "Paluma" armazenada a $-20^{\circ} \mathrm{C}$. Revista Brasileira de Fruticultura, v. 25, n. 3, p. 394-396, 2003.

BURDURLU, H. S.; KOCA, N.; KARADENIZ, F. Degradation of vitamin $\mathrm{C}$ in citrus juice concentrates during storage. Journal of Food Engineering, v. 74, n. 2, p. 211-216, 2006.

COSTA, M. C. O. et al. Storage stability of cashew apple juice preserved by hot fill and aseptic processes. Ciência e Tecnologia de Alimentos, v. 23, supl., p. 106-109, 2003.

DJILAS, S. M.; MILIC, B. L. J. Naturally occurring phenolic compounds as inhibitors of free radical formation in the Maillard reaction. In: LABUZA, T. P. et al. (eds). Maillard reaction in chemistry, food and health. Cambridge: Royal Society of Chemistry, 1994. p. 75-80.

FERNANDES, A. G. et al. Sucos tropicais de acerola, goiaba e manga: avaliação dos padrões de identidade e qualidade. Revista CERES, v. 53, n. 307, p. 302-308, 2006.

FILGUEIRAS, H. A. C.; ALVES, R. E.; MOURA, C. F. H. Cajá (Spondias mombim L.). In: ALVES, R. E.; FILGUEIRAS, H. A. C.; MOURA, C. F. H. (orgs). Caracterização de frutas nativas da América Latina. Jaboticabal: UNESP, 2000.

FRANCIS, F. J. Analysis of anthocyanins. In: MARKAKIS, P. (ed.). Anthocyanins as food colors. New York: Academic Press, 1982. p. 181-207.

FRANCIS, F. J. Food colorants: anthocyanins. Critical Reviews in Food Science and Nutrition, v. 28, n. 4, p. 273-314, 1989.

FREITAS, C. A. S. et al. Storage stability of acerola tropical fruit juice obtained by hot fill method. International Journal of Food Science and Technology, v. 41, n. 10, p. 1216-1221, 2006 a.

FREITAS, C. A. S. et al. Estabilidade dos carotenóides, antocianinas, vitamina $C$ presentes no suco tropical de acerola (Malpighia emarginata D.C.) adoçado envasado pelos processos hot fill e asséptico. Ciência e Agrotecnologia, v. 30, n. 5, p. 942-949, 2006 b.

HIGBY, W. K. A simplified method for determination of some aspects of the carotenoid distribution in natural and carotene-fortified orange juice. Journal of Food Science, v. 27, n. 1, p. 42-49, 1962.

JOHNSTON, C. S.; HALE, J. C. Oxidation of ascorbic acid in stored orange juice is associated with reduced plasma vitamin $\mathrm{C}$ concentrations and elevated lipid peroxides. Journal of the American Dietetic Association, v. 105, n. 1, p. 106-109, 2005.

MAGALHÃES, E. F. Estabilidade do suco tropical de manga (Mangifera indica L.) adoçado e envasado pelos processos hot fill e asséptico. Ceará, 2005. 171 p. Dissertação (Mestrado em Tecnologia de Alimentos) - Universidade Federal do Ceará - UFC.

MAIA, G. A. et al. Técnica aumenta tempo de conservação da goiaba. Revista de Ciência e Tecnologia da FUNCAP, v. 4, n. 1, p. 11-12, 2002.

MAIA, G. A.; SOUSA, P. H. M.; LIMA, A. S. Processamento de sucos de frutas tropicais. Fortaleza: UFC, 2007. 320 p. 
MANNHEIM, C. H.; MAVKIN, M. Shelf-life of aseptically bottled orange juice. Journal of Food Processing and Preservation, v. 5, n. 1, p. 1-6, 1981.

MARTIN, J. J. et al. Evolución quimica y organoleptica del zumo de naranja pasterizado. Alimentaria, v. 4, n. 261, p. 59-63, 1995.

MATIOLI, G.; RODRIGUEZ-AMAYA, D. B. Microencapsulação do licopeno com ciclodextrinas. Ciência e Tecnologia de Alimentos, v. 23, supl., p. 102-105, 2003.

POLYDERA, A. C.; STOFOROS, N. G.; TAOUKIS, P. S. Quality degradation kinetics of pasteurized and high pressure processed fresh Navel orange juice: Nutritional parameters and shelf life. Innovative Food Science \& Emerging Technologies, v. 6, n. 1, p. 1-9, 2005.

SAHARI, M. A.; BOOSTANI, F. M.; HAMIDI, E. Z. Effect of low temperature on the ascorbic acid content and quality characteristics of frozen strawberry. Food Chemistry, v. 86, n. 3, p. 357-363, 2004.

SARANTÓPOULOS, C. I. G. L.; OLIVEIRA, L. M.; CANAVESI, É. Alterações de alimentos que resultam em perda de qualidade. In: SARANTÓPOULOS, C. I. G. L.; OLIVEIRA, L. M.; CANAVESI, É. Requisitos de conservação de alimentos em embalagens flexíveis. Campinas: CETEA, 2001. p. 1-22. (cap. 1)

SAS INSTITUTE. SAS User's Guide. Version 9.1. Cary, 2006.

SELLAPPAN, S.; AKOH, C. C.; KREWER, G. Phenolic compounds and antioxidant capacity of Georgia-grown blueberries and blackberries. Journal of Agricultural and Food Chemistry, v. 50, n. 8 , p. $2432-2438,2002$.
SUNTORNSUK, L. et al. Quantitation of vitamin C content in herbal juice using direct titration. Journal of Pharmaceutical and Biomedical Analysis, v. 28, n. 5, p. 849-855, 2002.

UNITED STATES DEPARTMENT OF AGRICULTURE - USDA. Agricultural Research Service: Nutrient Data Laboratory. Washington, 2006. Disponível em: <www.nal.usda.gov/fnic/ foodcomp >. Acesso em: 17 jan. 2007.

VANNUCHI, H.; JORDÃO Jr., A. A. Vitaminas hidrossolúveis, In: OLIVEIRA, J. E. D.; MARCHINI, J. S. (orgs). Ciências nutricionais. Sarvier: São Paulo, 1998. p. 191-208.

VENDRAMINI, A. L.; TRUGO, L. C. Phenolic compounds in acerola fruit (Malpighia punicifolia, L.). Journal of the Brazilian Chemical Society, v. 15, n. 5, p. 664-668, 2004.

VIKRAM, V. B.; RAMESH, M. N.; PRAPULLA, S. G. Thermal degradation kinetics of nutrients in orange juice heated by electromagnetic and conventional methods. Journal of Food Engineering, v. 69, n. 1, p. 31-40, 2005.

WORLD CANCER RESEARCH FUND - WCRF. Food, nutrition and prevention of cancer: a global perspective. Washington, 1997. $670 \mathrm{p}$.

WORD CANCER RESEARCH FUNDATION AND AMERICAN INSTITUTE FOR CANCER RESEARCH - WTCR/AICR. Food, Nutrition and Prevention of Cancer: a Global Perspective. Washington: WTCR/AICR, 1997.670p.

ZIELINSKI, H.; KOZLOWSKA, H. Antioxidant activity and total phenolics in selected cereal grains and their different morphological fractions. Journal of Agricultural and Food Chemistry, v. 48, n. 6 , p. 2008-2016, 2000. 